\title{
Design of Underwater Acoustic Channel Model for OFDM Communication System
}

\author{
Naveen H, Chetan H, Sudatta Mohanty, Sangmesh Melinmani, Sreerama Reddy G M
}

\begin{abstract}
The paper describes the design of underwater acoustic channel model for OFDM communication system. The underwater acoustic channel considered includes multipath effect, attenuation loss, absorption loss, spreading loss and total noise due to thermal Noise, turbulence Noise, shipping Noise, wave Noise. The acoustic channel model used for OFDM Communication will have the noise effect due to attenuation loss and ambient noise (Total Noise). In this research work, In the underwater channel, Multipath parameters such as length, width and depth and attenuation parameters such as frequency, salinity, radial range, SPS and ambient noise parameters such as shipping factor, wave factor are considered in calculating total noise.
\end{abstract}

Keywords: Attenuation, Spreading, Absorption, Underwater Acoustic channel.

\section{INTRODUCTION}

Physical and chemical properties of seawater affect sound propagation. Due to spreading and absorption, an underwater acoustic signal will suffer attenuation. Furthermore, depending on channel geometry, multipath fading may occur and produce significant inter-symbol-interference (ISI) at the receiver hydrophone. For calculations of the Signal-to-Noise ratio (SNR) or Bit Error Rate (BER) estimation, it is then crucial to understand and establish a good channel model.

Discovering and exploring new environments is an important human endeavor, a motor for mankind's evolution. One vast environment which is still much unexplored is the underwater world. Crucial for its successful exploration are reliable communication systems.

The topic is complex and there are various difficulties in underwater communications, such as water chemical constitution, environmental variables, and the presence of various types of noise. A promising solution, which has been studied and implemented for communicating within this environment, is the use of acoustic waves for the transmission of signals. Electromagnetic waves usually are not considered as a solution for underwater communications

Revised Version Manuscript Received on April 12, 2019.

Naveen H, Department of Electronic and Communication, New Horizon College of Engineering, Bangalore, India

Chetan H, Department of Electronic and Communication, C M R Institute of Technology, Bangalore, India

Sudatta Mohanty, Department of Electronic and Communication, CMR Institute of Technology, Bangalore, India

Sangmesh Melinmani, Department of Electronics and Communication,DSCE, Bangalore, India

Dr.Sreerama Reddy G M, Department of Electronics and Communication, CBIT, Kolar, India because their attenuation is too high.

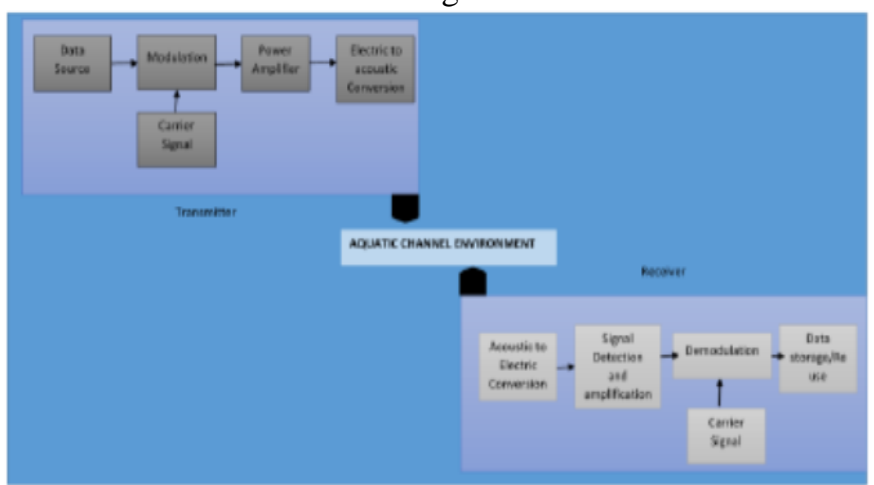

Figure.1. Top level Block Diagram of Underwater Acoustic Model

Acoustic waves appear as a good alternative, despite some associated negative aspects. For long communicating distances, an abrupt decay in pressure may occur, impairing the communication quality. This phenomenon may occur even for medium distances and it is dependent of the transmitting acoustic wave frequency.

Interest from the research community in the area of underwater communications has increased recently and this work aims to be a contribution to the development of the Underwater Acoustic Communication (UWAC) field.

\section{UNDERWATER CHANNEL CHARACTERISTICS}

The present work focuses on the aquatic channel software model for verification with real-world measurements. Fig. Below gives the aquatic channel block breakdown in several internal blocks.

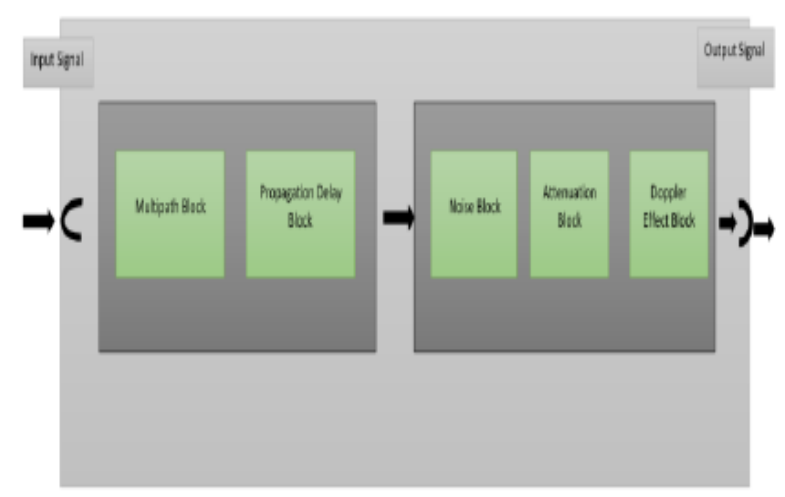

Figure 2. Aquatic Channel Model

Published By:

Blue Eyes Intelligence Engineering 


\section{Design Of Underwater Acoustic Channel Model For Ofdm Communication System}

\section{A. Spreading loss}

As the wave front moves outward from source, spreading loss occurs due to ever-increasing area covered by same amount of sound signal energy, and it is given by

PL spreading(r) = k X $10 \log (r)(d B)$

Where $\mathrm{r}$ is the range in meters and $\mathrm{k}$ is the spreading factor.

\section{B. Absorption Loss}

PL $\operatorname{absorption}(r, f)=10 \log (\alpha$ ( f )) $X$ r (dB)

$\alpha=$ Absorption Co-Efficient depends on Frequency

Absorption coefficient, $\alpha$, increases with increasing frequency and decreases as depth increases.

Effect of viscosity becomes significant with high frequencies above $100 \mathrm{kHz}$, whereas ionic relaxation effects of magnesium affect mid frequency range from $10 \mathrm{kHz}$ up to $100 \mathrm{kHz}$, and boric acid effects dominate at low frequencies, which go up to a few $\mathrm{kHz}$.

\section{Path loss}

Total path loss, or just path loss, is combined contribution of both spreading and absorption losses

$\operatorname{PathLoss}(r, f, d, t)=k * 10 \log (r)+\alpha(f, d, t) * r * 10^{3} \mathrm{db}$

\section{Ambient Noise}

For underwater channel four noise components are considered. There is thermal noise ( $\mathrm{Nth}(\mathrm{f}))$, that can be taken as additive white Gaussian noise that is always present in communications systems. Then, there is noise due to movement of waves $(\mathrm{Nw}(\mathrm{f}))$, making water medium unstable and varying static properties of channel. Another type of noise can be water movement caused by ship traffic (Ns(f)) and, finally, noise due to natural causes ( $\mathrm{Nt}(\mathrm{f})$ ), like turbulence caused by some storms or during rain events and presence of bubbles.

Turbulence Noise

$N t=10^{\frac{\pi(17-30 \log 10 f w}{10}} N t$

$$
\begin{aligned}
& \text { Shipping Noise } \\
& N s=10^{\frac{(f 0+[(z 0)(s-0.5)]+[(z 6) \log 10(f)]-[60 \log 10(f+0.03)] 0]}{10}} N_{\text {soff }}
\end{aligned}
$$

Wave Noise

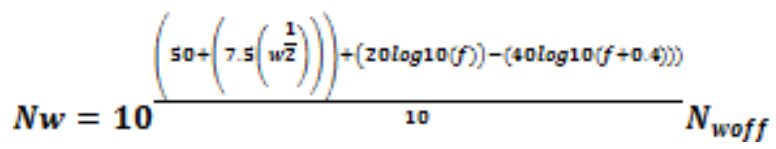

Thermal Noise

$N_{\text {th }}=10^{\frac{\pi-15+20 \log 100 f \mathbb{m}}{10}} N_{\text {thoff }}$

Total Noise $=\mathrm{N}_{\mathrm{t}}+\mathrm{N}_{\mathrm{s}}+\mathrm{N}_{\mathrm{w}}+\mathrm{N}_{\mathrm{th}}$

\section{E. Multipath}

Width of aquarium was in $\mathrm{x}$ axis, the length in the $\mathrm{y}$ axis, depth in the $\mathrm{z}$ axis, and these dimensions are represented by constants $\mathrm{W}, \mathrm{L}$ and $\mathrm{D}$, respectively.
$\mathrm{Rx} 1=\left[\mathrm{x}_{2}, \mathrm{y}_{2}, \mathrm{z}_{2}\right]$

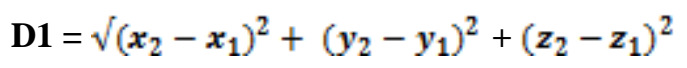

$\mathbf{R x} 2=\left[2 * W-\mathbf{x}_{2}, \mathbf{y}_{2}, z_{2}\right]$

D2 $=\sqrt{\left(2 * W-x_{2}-x_{1}\right)^{2}+\left(y_{2}-y_{1}\right)^{2}+\left(z_{2}-z_{1}\right)^{2}}$

$\mathbf{R x} 3=\left[-\mathbf{x}_{2}, \mathbf{y}_{2}, \mathbf{z}_{2}\right]$

D3 $=\sqrt{ }\left(-x_{2}-x_{1}\right)^{2}+\left(y_{2}-y_{1}\right)^{2}+\left(z_{2}-z_{1}\right)^{2}$

$\mathbf{R x} 4=\left[\mathbf{x}_{2}, \mathbf{y}_{2}, 2 * \mathbf{D}-\mathbf{z}_{2}\right]$

D4 $=\sqrt{\left(x_{2}-x_{1}\right)^{2}}+\left(y_{2}-y_{1}\right)^{2}+\left(2 * \mathrm{D}-z_{2}-z_{1}\right)^{2}$

$\operatorname{Rx5}=\left[\mathbf{x}_{2}, \mathbf{y}_{2},-\mathbf{z}_{2}\right]$

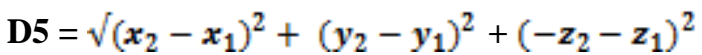

$\mathbf{R x 6}=\left[\mathbf{x}_{2}, 2 * L-y_{2}, z_{2}\right]$

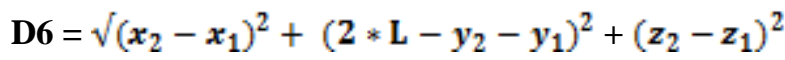

$\mathbf{R x} 7=\left[\mathbf{x}_{2},-\mathbf{y}_{2}, \mathbf{z}_{2}\right]$

D7 $=\sqrt{ }\left(x_{2}-x_{1}\right)^{2}+\left(-y_{2}-y_{1}\right)^{2}+\left(z_{2}-z_{1}\right)^{2}$

Absorption Coefficient is given by

$\alpha=\frac{0.106 \cdot f 1 \cdot f^{2} * e^{\frac{p h-8}{0.56}}}{f_{1}{ }^{2}+f^{2}}+\frac{0.52 \cdot\left(1+\frac{T}{43}\right) \cdot \frac{5}{45} * f 2 \cdot f^{2} * e^{\frac{-D}{6}}}{f_{2}{ }^{2}+f^{2}}+0.00049 * f^{2} * e^{\left(-\frac{T}{27}+\frac{D}{17}\right)}$

Absorption Loss for different path is given by
$\alpha 1=\frac{\alpha * D 1}{10^{5}}$
$\alpha 2=\frac{\alpha * D 2}{10^{5}}$
$\alpha 3=\frac{\alpha * D 3}{10^{5}} \quad \alpha 4=\frac{\alpha * D 4}{10^{5}}$
$\alpha 5=\frac{\alpha \cdot D 5}{10^{5}}$
$\alpha 6=\frac{\alpha * D 6}{10^{5}}$
$\alpha 7=\frac{\alpha \star D 7}{10^{5}}$

Spreading Loss and Total Path Loss for different path is given by

$$
\begin{aligned}
& s p 1=10 \log 10\left(\frac{\left(2 \cdot \text { ang } \cdot D 1^{2}\right)+d e}{d e}\right) \\
& P L 1=10^{\frac{(-\alpha 1-x p 1)}{10}} \\
& s p 2=10 \log 10\left(\frac{\left(2 \cdot \text { ang* } D 2^{2}\right]+d e}{d e}\right) \\
& P L 2=10^{\frac{(-\alpha z-s y z)}{10}} \\
& s p 3=10 \log 10\left(\frac{\left(2 \cdot \text { ang } \cdot D 3^{2}\right)+d e}{d e}\right) \\
& P L 3=10^{\frac{\left(-\alpha 3-x y^{3}\right.}{10}} \\
& s p 4=10 \log 10\left(\frac{\left(2 \cdot \text { ang* } D 4^{2}\right)+d e}{d e}\right) \\
& P L 4=10^{\frac{(-\alpha 4-3 p 4)}{10}} \\
& s p 5=10 \log 10\left(\frac{\left(2 \text { ang* } D 5^{2}\right)+d e}{d e}\right) \\
& P L 5=10^{\frac{\left(-\alpha 5-3 y^{5}\right.}{10}} \\
& s p 6=10 \log 10\left(\frac{\left(2 * \text { ang } * D 6^{2}\right)+d e}{d e}\right) \\
& P L 6=10^{\frac{(-\alpha 6-s p 6)}{10}}
\end{aligned}
$$


International Journal of Innovative Technology and Exploring Engineering (IJITEE)

ISSN: 2278-3075, Volume-8, Issue- 6S4, April 2019

$$
\begin{aligned}
s p 7= & 10 \log 10\left(\frac{\left(2 * \text { ang } * D 7^{2}\right)+d e}{d e}\right) \\
P L 7= & 10^{\frac{\left(-\alpha 7-3 p^{2}\right)}{10}} \\
& \text { de }=\pi * r r^{2} \text { if } \mathrm{r}<\lambda \quad \text { ang }=\pi / 2
\end{aligned}
$$

$r>\lambda \quad$ ang $=\operatorname{asin}(\lambda / d)$

Attenuation Loss

at $1=\frac{D 1}{C} * S P S \quad$ at $2=\frac{D 2}{C} * S P S \quad$ at $3=\frac{D 3}{C} * S P S$ at4 $=\frac{D 4}{C} *$ SPS $\quad$ at5 $=\frac{D 5}{C} *$ SPS $\quad$ at $6=\frac{D 6}{C} * S P S$ $a t 7=\frac{D 7}{C} * S P S$

\section{MATHEMATICAL MODEL}

\section{A. Multipath Parameters}

Let us consider $\mathrm{TX}=(\mathrm{x} 1, \mathrm{y} 1, \mathrm{z} 1)=(3,4,5)$ and $\mathrm{Rx}=$ $(\mathrm{x} 2, \mathrm{y} 2, \mathrm{z} 2)=(7,8,9)$ and aquarium dimension as Width $\mathrm{W}=20$ Length $=30$ Depth $=10$

D1 $=\sqrt{(7-3)^{2}+(8-4)^{2}+(9-5)^{2}=6.9283}$

$\mathrm{Rx} 2=[2 * 20-7,8,9]=[33,8,9]$

D2 $=\sqrt{(33-3)^{2}+(8-4)^{2}+(9-5)^{2}=30.52}$

$\operatorname{Rx3}=[-7,8,9]$

D3 $=\sqrt{ }(-7-3)^{2}+(8-4)^{2}+(9-5)^{2}=11.48$

$\mathrm{Rx} 4=[7,8,2 * 10-9]=[7,8,11]$

D4 $=\sqrt{(7}-3)^{2}+(8-4)^{2}+(11-5)^{2}=8.246$

$\operatorname{Rx5}=\left[\mathbf{x}_{2}, \mathbf{y}_{2},-\mathbf{z}_{2}\right]=[7,8,-9]$

D5 $=\sqrt{(7}-3)^{2}+(8-4)^{2}+(-9-5)^{2}=15.099$

$\operatorname{Rx6}=[7,2 * 30-8,9]=[7,52,9]$

D6 $=\sqrt{(7-3)^{2}}+(52-4)^{2}+(9-5)^{2}=48.33$

$\operatorname{Rx} 7=\left[\mathbf{x}_{2},-\mathbf{y}_{2}, \mathbf{z}_{2}\right]=[7,-8,9]$

D7 $=\sqrt{(7}-3)^{2}+(-8-4)^{2}+(9-5)^{2}=13.266$

B. Attenuation Parameters

Let us consider attenuation parameters, Frequency $\mathrm{f}=$ $1000 \mathrm{kHz}$, Radius $\mathrm{r}=1 \mathrm{~cm}$, Depth $\mathrm{D}=1$ Radius Reference rr $=10 \mathrm{~cm}$, Salinity $\mathrm{S}=1 \mathrm{ppt}$, Temperature $=30, \mathrm{Ph}=7.2 \mathrm{ppm}$, $\mathrm{SPS}=2.5 \mathrm{e}^{\wedge} 7=2741.58$

$f 1=0.78 * \sqrt{\frac{1}{35}} * e^{\frac{30}{26}}=0.4163$

$f 2=42 * e^{\frac{30}{17}}=245.27$

Cmetro $=\mathbf{1 5 3 5 . 2 7 5}$

$\mathrm{C}=$ Cmetro $* 100=\mathbf{1 5 3 5 2 7 . 5}$

$$
\lambda=\frac{c}{(f \cdot 1000)}=\mathbf{0 . 1 5 3 5 3}
$$

Absorption Co-Efficient

$\alpha=\frac{0.106 \cdot f 1 \cdot f^{2} * e^{\frac{P h-8}{0.56}}}{f_{1}^{2}+f^{2}}+\frac{0.52 *\left(1+\frac{T}{43}\right) * \frac{5}{45} * f 2 * f^{2} * e^{\frac{-D}{6}}}{f_{z}^{2}+f^{2}}+0.00049 * f^{2} * e^{\left(-\frac{T}{27}+\frac{D}{17}\right)}$

$$
=155.9422
$$

Absorption Loss :

$$
\begin{aligned}
& \alpha 1=\frac{\alpha * D 1}{10^{5}}=155.942 * 6.9283 / 10^{5}=0.010804 \\
& \alpha 2=\frac{\alpha * D 2}{10^{5}}=155.942 * 30.52 / 10^{5}=0.0475 \\
& \alpha 3=\frac{\alpha * D 3}{10^{55}}=155.942 * 11.48 / 10^{5}=0.0179 \\
& \alpha 4=\frac{\alpha * D 4}{10^{5}}=155.942 * 8.246 / 10^{5}=0.01285 \\
& \alpha 5=\frac{\alpha * D 5}{115}=155.942 * 15.099 / 10^{5}=0.023547 \\
& \alpha 6=\frac{\alpha * D 6}{10^{5}}=155.942 * 48.33 / 10^{5}=0.07822 \\
& \alpha 7=\frac{\alpha * D 7}{10^{5}}=155.942 * 13.266 / 10^{5}=0.029423
\end{aligned}
$$

Spreading Parameters :

$$
\begin{array}{ccc}
\text { de }=\pi * r r^{2}=314.15 & \text { if } r<\lambda \quad \text { ang }=\pi / 2 \\
d=2 * r=2 * 1=2 \mathrm{~cm} & r>\lambda & \text { ang }=\operatorname{asin}(\lambda / d) \quad \text { ang } \\
=\operatorname{asin}(0.15353 / 2)=0.07684 & &
\end{array}
$$

Spreading Loss

$$
s p 1=10 \log 10\left(\frac{\left(2 * \text { ang* } D 2^{2}\right)+d e}{d e}\right)=10 \log 10\left(\frac{\left(2 * 0.07684 * 6.9283^{2}\right)+314.15}{314.15}\right)
$$$$
=0.1008
$$$$
\operatorname{sp2}=10 \log 10\left(\frac{\left(2 * \text { ang* } D 2^{2}\right)+d e}{d e}\right)=
$$$$
10 \log 10\left(\frac{\left(2 \cdot 0.07684 \cdot 30.52^{2}\right)+314.15}{314.15}\right)=1.6313
$$$$
s p 3=10 \log 10\left(\frac{\left(2 * \text { ang* } D 3^{2}\right)+d e}{d e}\right)=
$$$$
10 \log 10\left(\frac{\left(2 * 0.07684 * 11.48^{2}\right)+314.15}{314.15}\right)=\mathbf{0 . 2 7 1 7 5}
$$$$
s p 4=10 \log 10\left(\frac{\left(2 \text { ang* } D 4^{2}\right)+d e}{d e}\right)=
$$$$
10 \log 10\left(\frac{\left(2 * 0.07684 \cdot 8.246^{2}\right)+314.15}{314.15}\right)=\mathbf{0 . 1 4 2 1 1}
$$$$
s p 5=10 \log 10\left(\frac{\left(2 * \text { ang* D } 55^{2}\right)+d e}{d e}\right)=10 \log 10\left(\frac{\left(2 \cdot 0.07684 \cdot 15.099^{2}\right)+314.15}{314.15}\right)
$$

$$
\begin{aligned}
& =0.45922 \\
& s p 7=10 \log 10\left(\frac{\left(2 * \text { ang* } D 7^{2}\right)+d e}{d e}\right)=
\end{aligned}
$$

Total Path Loss

$$
\begin{aligned}
& P L 1=10^{\frac{\left(-\alpha 1-3 p^{1}\right)}{10}}=10^{\frac{(-0.010804-0.1008)}{10}}=0.9746 \\
& P L 2=10^{\frac{(-\alpha z-5 p 2)}{10}}=10^{\frac{(-0.0475-1.6313)}{10}}=0.6793 \\
& P L 3=10^{\frac{(-\alpha 3-373)}{10}}=10^{\frac{(-0.0179-0.27175)}{10}}=0.9354 \\
& P L 4=10^{\frac{(-\alpha 4-3 p 4)}{10}}=10^{\frac{(-0.01285-0.14211)}{10}}=0.9649 \\
& P L 5=10^{\frac{(-\alpha 5-3 p 5)}{10}}=10^{\frac{(-0.023547-0.459 z z)}{10}}=0.8948 \\
& P L 6=10^{\frac{(-\alpha 6-3 y 6)}{10}}=10^{\frac{(5-0.078 z z-3.4845)}{10}}=0.4402 \\
& P L 7=10^{\frac{(-\alpha 7-3 p D)}{10}}=10^{\frac{(-0.029423-0.69722)}{10}}=0.8459
\end{aligned}
$$

Attenuation Loss :

$$
\begin{aligned}
& \text { at } 1=\frac{D 1}{C} * S P S=\frac{6.92}{153527.5} * 2741.5=0.1235 \\
& \text { at } 2=\frac{D 2}{C} * S P S=\frac{150.52}{153527.5} * 2741.5=0.42143 \\
& a t 3=\frac{D 3}{C} * S P S= \\
& \frac{11.48}{153527.5} * 2741.5= \\
& \mathbf{0 . 0 8 1 4 4 3} \\
& \text { Published By: } \\
& \text { Blue Eyes Intelligence Engineering } \\
& \text { \& Sciences Publication }
\end{aligned}
$$




$$
\begin{aligned}
& \text { at4 }=\frac{D 4}{C} * S P S=\frac{8.24}{153527.5} * 2741.5=\mathbf{0 . 0 2 3 5 3 5} \\
& \text { at5 }=\frac{D 5}{C} * S P S=\frac{15.09}{153.527 .5} * 2741.5=\mathbf{0 . 1 4 5 9 2} \\
& \text { at6 }=\frac{D 6}{C} * S P S=\frac{4833}{153527.5} * 2741.5=\mathbf{0 . 7 7 1 9 7} \\
& \text { at7 }=\frac{D 7}{C} * S P S=\frac{13.26}{153527.5} * 2741.5=0.21321
\end{aligned}
$$

\section{C.Ambient Noise Parameters}

Let us consider the shipping Factor $=1$ Wind Speed $=10$ MEV $=1$, Ntoff $=1$, Nsoff $=1$, Nwoff $=1$, Nthoff $=1$

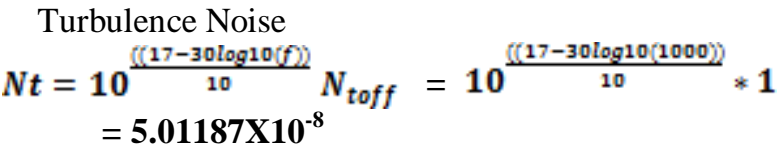

Shipping Noise

$$
\begin{aligned}
& N s=10 \frac{(f(a 0+[20(s-0.5)]+[(z 6) \log 10(f)]-[60 \log 10(f+0.03)] 0}{10} N_{s o f f} \\
& =10^{\frac{\operatorname{ff}(40+[20(1-0.5)]+[26 \log 10(1000)]-[60 \log 10(1000+0.03)]}{10}} * 1 \\
& =6.309 \times 10^{-6}
\end{aligned}
$$

Wave Noise

$$
N w=10 \frac{\left(50+\left(75\left(w^{\frac{1}{2}}\right)\right)\right)+(20 \log 10(f))-(10 \log 10(f+0.40)}{10} N_{\text {Woff }}
$$

$$
\begin{aligned}
& =10 \frac{\left(50+\left(75\left(10 \frac{1}{2}\right)\right)\right)+(20 \log 10(1000)]-(40 \log 10(1000+0.4200)}{10} * 1 \\
& =23.49
\end{aligned}
$$

Thermal Noise

$$
\begin{aligned}
& N_{\text {th }}=10^{\frac{\text { It-15+20log10/fin }}{10}} N_{\text {thoff }} \\
& =10^{\frac{01-15+20 \log 10(1000)}{10}} * 1 \\
& =31622.7766
\end{aligned}
$$

Total Ambient Noise $=\mathbf{N}_{\mathbf{t}}+\mathbf{N}_{\mathrm{s}}+\mathbf{N}_{\mathrm{w}}+\mathbf{N}_{\mathrm{th}}=\mathbf{3 1 6 4 7 . 2 7 3 7}$

\section{RESULT AND ANALYSIS}

Below section provides the Graphical user interface (GUI) developed for Underwater Acoustic Channel Model considering Multipath Parameters, Attenuation parameters and Ambient Noise Parameters.

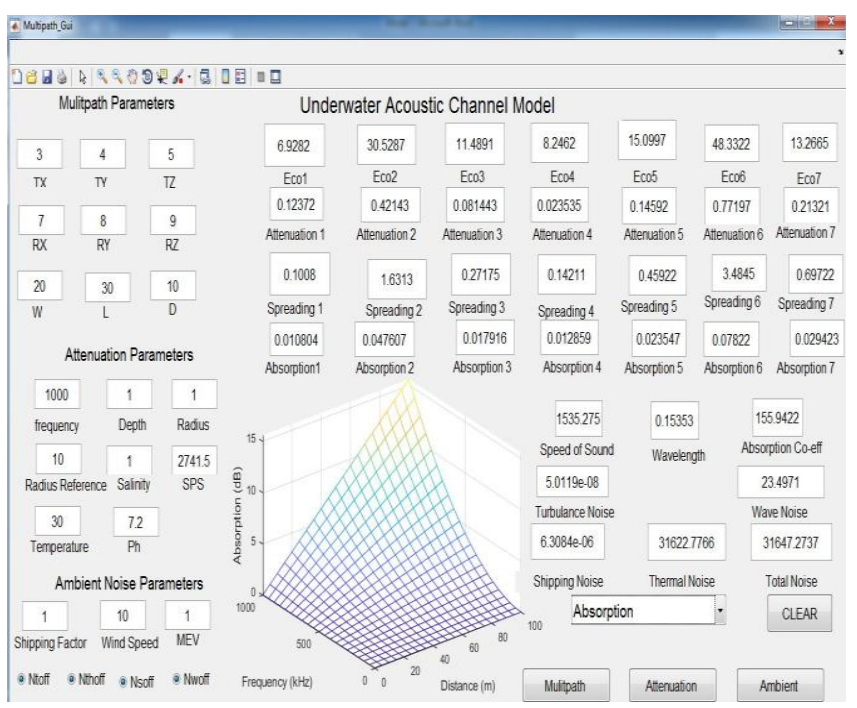

Figure 3. Underwater Acoustic Channel Model GUI with Absorption loss

Figure 3 shows MATLAB GUI for Underwater acoustic channel mode with absorption loss variation with frequency and distance $(\mathrm{m})$.

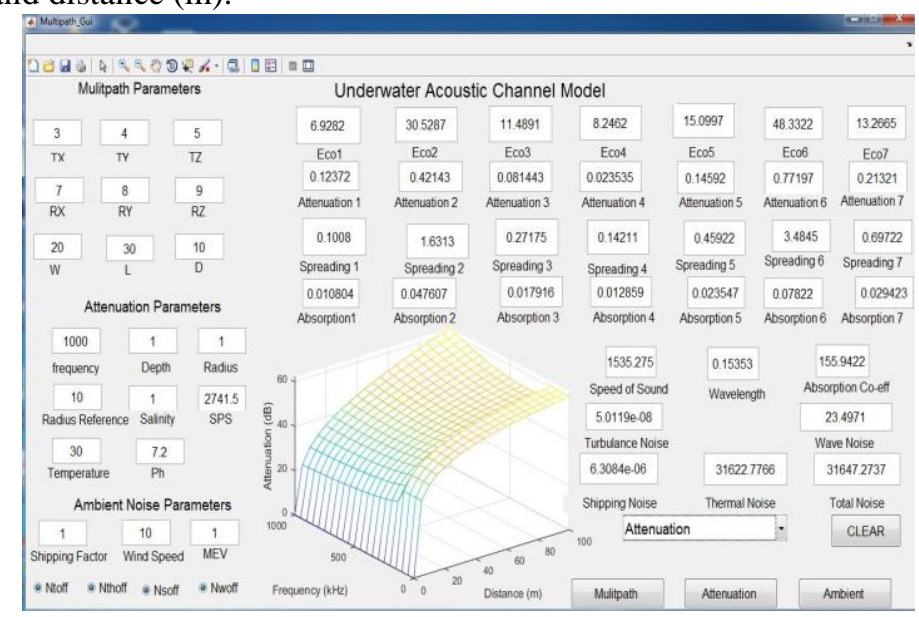

Figure 4: Underwater Acoustic Channel Model GUI with Attenuation loss

Figure 4 shows MATLAB GUI for Underwater acoustic channel mode with Attenuation loss variation with frequency and distance $(\mathrm{m})$.

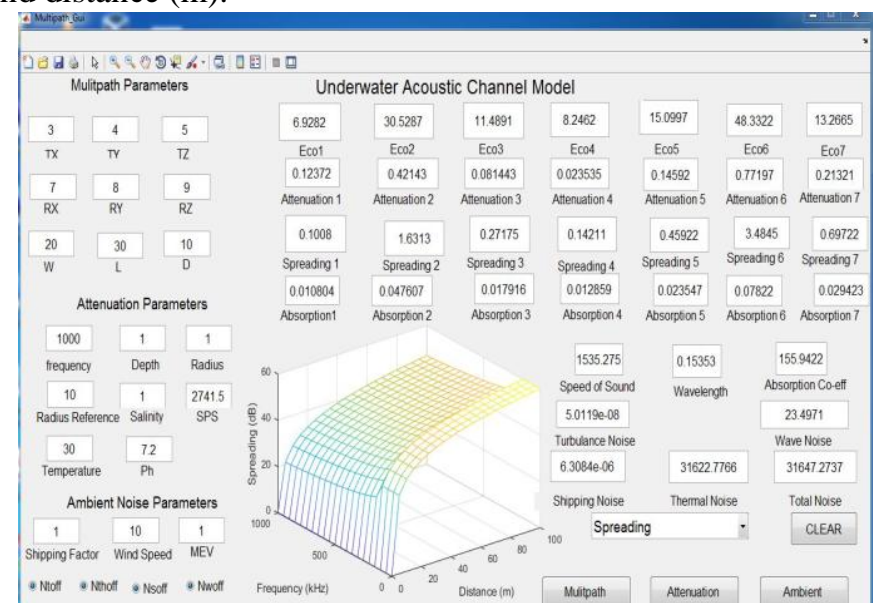

Figure 5: Underwater Acoustic Channel Model GUI with spreading loss

Published By. 


\section{CONCLUSION}

MATLAB GUI for Underwater acoustic channel model is been achieved in the research work. Underwater channel characteristics like Multipath parameters, Attenuation parameters and ambient noise Parameters are considered in modeling the Aquatic channel. GUI results provide with the total noise due to ambient noise parameters such as wave noise, thermal noise, turbulence noise and shipping noise. Attenuation loss is due to spreading loss and absorption loss and it is recorded for different path. Multipath distance is calculated by considering the aquarium dimension of specified Width, Length and height between a transmitter and receiver. Calculated ambient noise and attenuation loss values will be used as underwater acoustic channel in OFDM Communication system and results will be compared with OFDM Communication system in AWGN Channel.

\section{REFERENCES}

1. Naveen H, Dr.Sreerama Reddy G M, "Performance analysis of FFT based OFDM and DWT based OFDM for Underwater Acoustic Communication", Indian Journal of Science and Technology, Vol 10(36), September 2017.

2. Naven H, Dr.Sreerama Reddy G M, 'High Speed OFDM based Image transmission system for Remotely Operated Underwater Vehicle (ROV)', International Journal of Pure and Applied Mathematics, Vol 117, No.20, 2017, pp 959-970

3. Diogo Mendes, A MATLAB/Simulink model to evaluate underwater acoustic. Masters thesis, Universidade do Minho, Portugal, December (2011)

4. Kalangi Pullarao Prasanth. Modelling and simulation of an underwater acoustic communication channel. Masters thesis, University of applied sciences Bremen, Germany, (2004).

5. M. Stojanovic. Recent advances in high-speed underwater acoustic communications. Oceanic Engineering, IEEE Journal of, 21(2):125136, (1996)

6. Dario Pompili and Ian F. Akyildiz. Overview of networking protocols for underwater wireless communications. Comm. Mag., 47(1):97102, January (2009).V. K. Singh, "Discrete wavelet transform based image compression", International Journal of Remote Sensing, 2014.

7. as Kapoor, R., Gupta, R., Kumar, R. et al. Wireless Netw (2018) New scheme for underwater acoustically wireless transmission using direct sequence code division multiple access in MIMO systems 12 May 2018 ISSN:-1572-8196

8. Veena MB, Shanmukha Swamy MN. Performance analysis of DWT based OFDM over FFT based OFDM and implementing on FPGA. International Journal of VLSI design \& Communication Systems (VLSICS). 2011 September; 2:3.

9. Baosheng Li, Shengli Zhou, Milica Stojanovic, Lee Freitag, and Peter Willett. Multicarrier Communication over Underwater Acoustic Channels with Non uniform Doppler Shifts. IEEE Journal Of Oceanic Engineering. 2008

10. Yimin Shi, Zhankui Cao, Haichao Wei, Sihai Zhang, Wuyang Zhou. DSP Implementation of OFDM-based Underwater Acoustic Communication Transceiver IEEE. 2014.

11. Marcelo J. Weinberger, Senior Member, IEEE, Gadiel Seroussi, Fellow, IEEE, and Guillermo Sapiro, Member, IEEE, "The LOCO-I Lossless Image Compression Algorithm: Principles and Standardization into JPEG-LS",IEEE transactions on Image Processing, vol. 9, no. 8, August 2000.

12. Liu, Q., Chen, X., Liu, X., \& Linge, N. (2016). CACA-UAN: A context-aware communication approach based on the underwater acoustic sensor network. In International conference on cloud computing and security (pp. 37-47). Berlin: Springer.

13. Jiang, Z. (2008). Underwater acoustic networks-issues and solutions. International Journal of Intelligent Control and Systems, 13(3), $152-161$.
14. Poor, H. V., \& Tanda, M. (2002). Multiuser detection in flat fading non-Gaussian channels. IEEE Transactions on Communications, 50(11), 1769-1777.

15. Francois, R. E., \& Garrison, G. R. (1982). Sound absorption based on ocean measurements. Part II: Boric acid contribution and equation for total absorption. The Journal of the Acoustical Society of America, 72(6), 1879-1890.

16. Singer, A. C., Nelson, J. K., \& Kozat, S. S. (2009). Signal processing for underwater acoustic communications. IEEE Communications Magazine, 47(1), 90-96.

17. Cui, J. H., Kong, J., Gerla, M., \& Zhou, S. (2006). The challenges of building mobile underwater wireless networks for aquatic applications. IEEE Network, 20(3), 12-18.

18. Tao, J., Zheng, Y. R., Xiao, C., Yang, T. C., \& Yang, W. B. (2010). Channel equalization for single carrier MIMO underwater acoustic communications. EURASIP Journal on Advances in Signal Processing, 2010(1), 281769.

19. Iglesias, A. Song, J. Garcia-Frias, M. Badiey \& G. R. Arce (2011). Image transmission over the underwater acoustic channel via compressive sensing. In 45th annual conference on information sciences and systems, Baltimore, 23-25 March 2011 (pp. 1-6).

20. Hwang, S.-J., \& Schniter, P. (2007). Efficient communication over highly spread underwater acoustic channels. International Conference on Mobile Computing and Net-working, Montreal, 9-14, 11-18. 